Sci. Journal Impact

Factor: 6.1 (2018)

ICV: 90.90 (2018)

cc) (i) (8)

Copyright@IJCRR

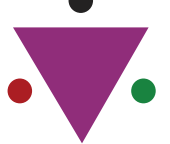 \\ IJCRR \\ Section: Healthcare \\ A Comparison of Overlapping Effects of Therapeutic Interventions to Improve Upper Limb Spasticity and Function in Stroke Patients}

\section{Jung-Ho Lee}

Professor, Department of Physical Therapy, School of Medical \& Public Health, Kyungdong University, Republic of Korea

\title{
ABSTRACT
}

Introduction: Stroke is a cerebrovascular disease that involves cerebral haemorrhage caused by the rupture of blood vessels and cerebral infarction due to blood clots and emboli, which result in neurological symptoms and disorders. Even though cerebrovascular diseases do not end up in death, they leave serious severe aftereffects in most cases, thus inflicting considerable social and economic losses.

Aim and Objective: The present study aimed to investigate the overlapping effects of low-extracorporeal shock wave therapy and proprioceptive neuromuscular facilitation treatment on stroke patients. In this study, 24 stroke patients who were randomly divided into an experimental group $(n=12)$, who would receive extracorporeal shock wave therapy before proprioceptive neuromuscular facilitation treatment, and a control group $(n=12)$, who would not receive extracorporeal shock wave therapy before proprioceptive neuromuscular facilitation treatment.

Result: This study used the modified Ashworth scale to assess stroke patients' spasticity. Korean version of the Fugl-Meyer assessment, the Korean version of the Modified Barthel index, and action research arm test are an evaluation item for measuring the function. In a comparison of the therapeutic effects on the two groups following the modified Ashworth scale and the Korean version of the Fugl-Meyer assessment, no statistically significant differences were found between the two groups. However, the Korean version of the Modified Barthel index and the action research arm test showed significant differences between groups.

Conclusion: In conclusion, the overlapping effect of proprioceptive neuromuscular facilitation treatment and extracorporeal shock wave therapy improved the upper limb function of stroke patients.

Key Words: Overlapping effect, Stroke, Function, Rehabilitation

\section{INTRODUCTION}

Stroke is a cerebrovascular disease that involves cerebral haemorrhage caused by the rupture of blood vessels and cerebral infarction due to blood clots and emboli, which result in neurological symptoms and disorders. Even though cerebrovascular diseases do not end up in death, they leave serious severe aftereffects in most cases, thus inflicting considerable social and economic losses.

The most common cause of cerebral haemorrhage is adult diseases, such as hypertension. The long-term exposure of capillary vessels, which supply oxygen and nutrients to brain tissues, to hypertension causes the deformation of blood vessels and excessive pressure increases. Patients with diabetes or hyperlipidemia are particularly susceptible to cerebral haemorrhage. They experience bleeding even at a minor blood pressure rise because their blood vessels cannot withstand it. Another cause of cerebral haemorrhage is bleeding due to the ageing of blood vessels or diseases themselves. One of its common subarachnoid haemorrhage caused by a ruptured cerebral aneurysm. Occasionally, haemorrhage also occurs due to cerebral arteriovenous malformations. Cerebral infarction refers to the occurrence of brain damage resulting from ischemia in closed blood vessels in the brain. This disease is classified as cerebral thrombosis and cerebral embolism. When blood vessels are clogged or compensation by collateral pathways is insufficient due to cerebral infarction, brain tissue cells undergo apoptosis, leading to permanent brain damage.

Stroke generally causes hemiplegia in the side opposite the brain damage, thereby exhibiting impairment or disabilities. Although hemiplegic patients show a highly effective

\section{Corresponding Author:}

Dr. Jung-Ho Lee, Professor, Department of Physical Therapy, School of Medical \& Public Health, Kyungdong University, Republic of Korea. Email: ljhcivapt@naver.com

ISSN: 2231-2196 (Print) ISSN: $0975-5241$ (Online)

Received: 20.06 .2020

Revised: 22.07 .2020

Accepted: 10.08 .2020

Published: 22.08.2020 
recovery of their lower limb function, their recovery of upper limb function lags far behind. Also, over $50 \%$ of patients who developed hemiplegia after a stroke experience weakened upper limb function on the affected side for a long period or permanently and are unable to use their affected upper limbs in daily living. The most typical problem of stroke is hemiplegia, and most hemiplegic patients exhibit abnormal motor patterns. These abnormal patterns mainly include the persistence of primitive reflexes, flexor synergy patterns, extensor synergy patterns, abnormal muscle tension, stiffening or hypertonicity, associated reactions, and associated movements.

Defects in upper limb function may be one of the most general neurological disorders that appear after a stroke. Although most of the upper limb function is restored within the first three months after stroke onset, clinical studies have reported that patients' performance of functional upper limb tasks, which include spontaneous movements of the shoulder joint, elbow joint, fingers, and wrist, is improved until one year after stroke onset. The functional recovery of upper limbs after a stroke, which is closely correlated with the ability to perform activities of daily living (ADLs) and the ability to perform independent ADLs, is handled importantly in the rehabilitation process. Movements in daily life are formed by their interactions with the nervous and muscular systems and the environment. Any damage to these areas causes abnormal movements in the human body.

Proprioception, which influences the nervous and muscular systems that control one's ability to perform ADLs, is used as basic information for the senses of balance and vibration. It also enables one to maintain the correct posture during every moment through feedback. Besides, proprioception controls the body to ensure its proper movements and affects the quality of motor control, including movement speed, sequential motions of several joints during a movement, and the act of holding a moving object. Moreover, proprioception provides the central nervous system with information about the perception of the body's location or posture, the angle and angular speed of every joint involved in movements on each plane, and the ratios of each joint's movements. It also plays the largest role in maintaining joint dynamic stability, helps induce normal movements, and protects joints safely from external damage.

Clinically, proprioception is an important element in the evaluation and treatment of patients with neurological problems; declines in proprioceptive function reduce one's ability to control postures, retain protective reflexes and joints' motor functions, and cope with external perturbations through balance, thereby causing problems in upper limb and ambulatory functions. Impaired proprioception frequently occurs in stroke patients and delays the recovery and enhancement of their motor skills. Even patients who have properly recov- ered their muscular strength after a stroke experience multiple disorders in performing ADLs if their proprioceptive damage persists.

Most stroke patients experience degradations in their abilities to control balance due to muscle tone changes, sensory defects, postural response abnormalities, motion range limitations, and cognitive decline. They also find it difficult to move their weight toward the affected side or maintain their centre of gravity; they cannot control their posture when performing functional motions. For this reason, balance control training is an important goal in the rehabilitation of stroke patients. Besides, the weakening of muscular strength is a factor that constrains the functional rehabilitation of stroke patients. It undermines functional activities and endurance, necessitates assistance in ADLs, and makes it difficult to change postures independently. This muscle weakening following a stroke stems from declines in the number of mobilizable motor units as a result of reduced descending information inputs; it also reduces the firing rate and impairs the harmony of motor units.

Existing rehabilitation programs conducted to improve stroke patients' upper limb function on the affected side include proprioceptive neuromuscular facilitation (PNF), neurodevelopmental treatment (NDT), constraint-induced movement therapy (CIMT), cognition-perception training, and electrical stimulation therapy. The leading types of electrical stimulation therapy are functional electrical stimulation therapy, extracorporeal shock wave therapy (ESWT), and transcutaneous electrical nerve stimulation. These treatments are easily applicable in clinical practice because they can be accurately aimed at the areas to be treated and quantitatively control the level of treatment. ESWT was first used to decompose stones in the kidney and the bile duct in 1976 but has since been applied as a new treatment technique for various orthopaedic diseases. ESWT restores damaged body regions by controlling the microenvironment through the exposure of lesion sites to shockwaves, which can destroy damaged tissues and cells while facilitating neovascularization and increasing local growth factors.

The therapeutic principle of ESWT is to generate physical and chemical changes in cell membranes by applying energy within an unharmful range to the human body and accurately exposing legion sites to shockwaves. ESWT produces positive effects when applied to musculoskeletal diseases, such as calcific tendinitis, tendonitis, plantar fasciitis, and epicondylitis, which are not easily cured with existing conservative therapies. At present, it is also used to reduce spasticity, control muscle tone, and control and treat other neurological disorders. Stroke patients' improvement in upper limb function serves as an important indicator of their functional recovery and an essential element in performing ADLs. Therefore, the present study aimed to investigate the overlapping effects of 
ESWT and PNF on stroke patients' spasticity reduction and improvement in upper limb function.

\section{RESEARCH METHODS}

\section{Subjects}

In this study, 24 stroke patients who were under hospital treatment at a rehabilitation hospital were selected as subjects. They were randomly divided into an experimental group ( $\mathrm{n}=12)$, who would receive ESWT before PNF, and a control group $(\mathrm{n}=12)$, who would not receive ESWT before PNF. The subjects voluntarily participated in the study after being fully informed of its purpose and contents, experimental procedures, and stability. They also submitted written participation consent before the experiment. All experimental procedures were performed following the Declaration of Helsinki.

The following individuals were selected as subjects: (1) Those who were diagnosed with a stroke at least six months before the study; (2) scored 24 points or more in the Korean mini-mental state examination (MMSE-K), which ensured their ability to understand the study's purpose and respond to verbal orders; (3) did not have visual or auditory disorders; (4) had no allergic responses to electrical stimulations; and (5) understood with their caregivers the study's purpose and agreed to participate in the study.

The following individuals were excluded from this study: (1) Those who were participating in regular exercises or other exercise programs; (2) had cerebellar disorders; (3) had other diseases that can affect their upper limb activities; (4) were under medication for spasticity reduction; or (5) had cardiovascular, kidney, liver, or cognitive disorders.

Both the experimental and control groups underwent proprioceptive neuromuscular facilitation treatment for $30 \mathrm{~min}$ utes, but the experimental group received ESWT in the upper limb for about 10 minutes before PNF. In the study, a pre-test evaluation was performed before the start of the therapeutic interventions, and a post-test evaluation was conducted after the final treatment. Both groups underwent their respective therapeutic interventions 18 times: three times a week for six weeks (Figure 1).

\section{Extracorporeal shock wave therapy}

ESWT was applied to the myotendinous junction in the affected upper limb to reduce the upper limb's spasticity and improve its function. Specifically, it was administered to an area $1 \mathrm{~cm}$ below the medial epicondylitis (origin of hand and wrist flexors), the still point (tuberosity of radius) of the biceps brachii (flexor of the elbow and shoulder joints), and the furrow between the tubercles of the humerus. The ESWT devices Optimus and Salus Talent 3 (Korea), which generate shock waves, were employed to apply ESWT to each area.

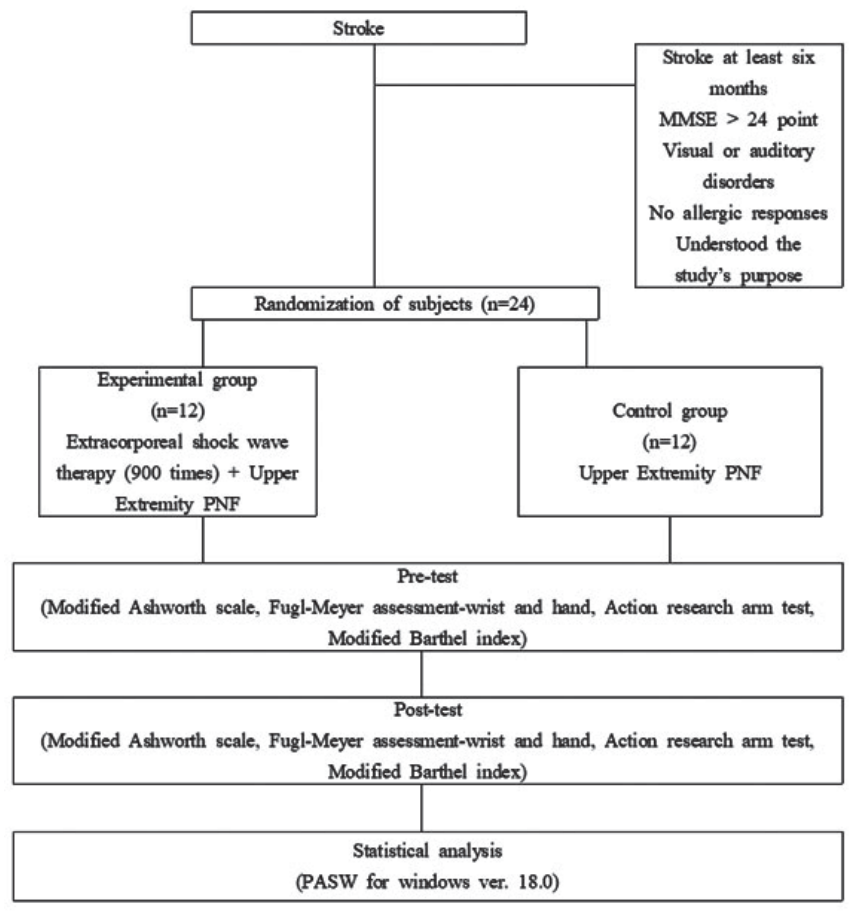

Figure 1: Flow chart for proprioceptive neuromuscular facilitation treatment.

To perform the ESWT treatment effectively, the patients were given a chair with a backrest for comfortable sitting. ESWT was applied when each patient extended the elbow joint up to the range that they could achieve through passive assisted exercises. For each area, the same shock wave stimulation was applied to the myotendinous junction 300 times, which translated into a total of 900 times for all areas, at a frequency of $2 \mathrm{~Hz}$ and power level of 1 . A one-minute break was provided after the treatment of each area.

\section{Proprioceptive neuromuscular facilitation}

Among the several types of PNF was used as general physical therapy for both groups in this study. The chopping pattern is an upper limb PNF pattern wherein the subject assumes the hook-lying position (bending the hip and knee joints in the supine position). The leading affected arm keeps the flexionadduction-external rotation of the shoulder joint, anterior elevation of the shoulder blade, extension-supination of the elbow joint, and flexion-radial deviation of the wrist.

Each subject held their left wrist in the unaffected arm during the flexion-adduction-external rotation of the shoulder joint. Then, they performed the chopping pattern (extensionadduction-internal rotation of the shoulder joint, posterior depression of the shoulder blade, extension-pronation of the elbow joint, and extension-ulnar deviation of the wrist) according to the researcher's instructions. Meanwhile, the 
extension-adduction-internal rotation pattern is an upper limb PNF pattern in which the subject assumes the hooklying position. In this position, each subject was instructed to place the affected arm at 11 o'clock, comfortably place the opposite arm by the trunk, and perform the upper limb extension-adduction-internal rotation pattern. A one-minute break was given after 10 rounds of performance to prevent muscle fatigue in the subjects.

\section{Assessment methods}

This study employed the modified Ashworth scale (MAS) to assess stroke patients' spasticity. The MAS measures the spasticity of patients through passive exercises based on six phases. Its scoring is as follows in Table 1.

\section{Table 1: Six Phase scoring for MAS Measures}

\begin{tabular}{|c|c|}
\hline Phases & MAS measures \\
\hline Grade o & No increase in muscle tone. \\
\hline Grade 1 & $\begin{array}{l}\text { A slight increase in muscle tone (minimal resist- } \\
\text { ance at the end of the range of motion (ROM) } \\
\text { when the subject holds or releases, or performs } \\
\text { flexion and extension of the affected region). }\end{array}$ \\
\hline Grade 1+ & $\begin{array}{l}\text { A slight increase in muscle tone (minimal resist- } \\
\text { ance at } 1 / 2 \text { or less of the ROM). }\end{array}$ \\
\hline Grade 2 & $\begin{array}{l}\text { A more visible increase in muscle tone at most } \\
\text { of the ROM but still easy movements. }\end{array}$ \\
\hline Grade 3 & $\begin{array}{l}\text { A definite increase in muscle tone that makes } \\
\text { movements difficult. }\end{array}$ \\
\hline Grade 4 & $\begin{array}{l}\text { Rigidity during flexion and extension that } \\
\text { makes passive movements impossible. }\end{array}$ \\
\hline
\end{tabular}

In this study, the MAS was measured during the extension of the affected elbow joint, and the mean value of three measurements was adopted as the measured value.

The Korean version of the Fugl-Meyer assessment (K-FMA) is a technique for the quantitative evaluation of stroke patients' functional recovery levels based on the phases of motor function recovery after a stroke. Its total score is 226 points in six domains: 66 points for the upper limb, 34 points for the lower limb, 14 points for balance, 24 points for sensation, 44 points for passive joint motions, and 44 points for pain. Each item is scored 0 to 2 points; a higher score indicates better motor control. In this study, the upper limb's ability to perform movements was assessed using scores for the wrist (10 points) and the hand (14 points), which are the FMA's items for the distal upper limb.

The action research arm test (ARAT) is an evaluation item for measuring the upper limb's function. It consists of 19 items with a perfect score of 57 points. It has four subdomains: holding (18 points), grabbing (12 points), picking up (18 points), and gross motion ( 9 points).

In this study, the Korean version of the Modified Barthel index (K-MBI) was used to assess stroke patients' ADLs. It evaluates movements based on five stages, from stage 1 (0 points), which refers to the inability to perform tasks, to stage 5, which means complete independence. A patient's higher level of independence results in varied scores at a higher stage. Its total score is 100 points based on a total of 10 items: personal hygiene, bathing, eating, toilet, going up and downstairs, dressing, bowel and bladder control, ambulation (wheelchair), and chair and bed transfer.

For every assessment, a pre-test was conducted before the therapeutic interventions, and a post-test was conducted after the final therapeutic intervention.

\section{STATISTICAL ANALYSIS}

For statistical data processing, mean \pm standard deviation values were derived from descriptive statistics using SPSS version 18.0, and an independent t-test was conducted to identify differences between the groups. A paired t-test was conducted to compare each group's changes before and after the experiment. The statistical significance level for all the data was set at $\mathrm{P}<0.05$.

\section{RESULTS OF STUDY}

\section{Modified ashworth scale (mas)}

In terms of the MAS score, the experimental and control groups had no statistically significant differences before and after the experiment $(\mathrm{P}>0.05)$ as shown in Table 2 . In a comparison of the therapeutic effects on the two groups following the intervention, no statistically significant differences were found between the two groups $(\mathrm{P}>0.05)$.

Table 2: Comparison of MAS scores between groups

\begin{tabular}{|c|c|c|c|c|c|c|}
\hline & \multicolumn{2}{|c|}{ Experimental group } & \multirow[t]{2}{*}{$\mathbf{p}$} & \multicolumn{2}{|c|}{ Control group } & \multirow[t]{2}{*}{$\mathbf{p}$} \\
\hline & Pre-test & Post-test & & Pre-test & Post-test & \\
\hline \multirow{3}{*}{$\begin{array}{l}\text { MAS } \\
\text { pre-post }\end{array}$} & $2.14 \pm 0.87$ & $1.82 \pm 0.83$ & 0.235 & $2.31 \pm 0.75$ & $2.18 \pm 0.71$ & 0.316 \\
\hline & \multirow{2}{*}{\multicolumn{2}{|c|}{$0.47 \pm 0.08$}} & & \multirow{2}{*}{\multicolumn{2}{|c|}{$0.25 \pm 0.07$}} & \\
\hline & & & 0.298 & & & \\
\hline
\end{tabular}

$<0.05$, MAS: modify Ashworth scale 


\section{Korean version of the fugl-meyer assessment (K-FMA)}

In terms of the FMA's wrist score, each group exhibited a statistically significant difference before and after the experiment $(\mathrm{P}<0.05)$. No statistically significant differences were observed between the two groups in terms of the therapeutic ef- fects following the intervention $(\mathrm{P}>0.05)$ as shown in Table 3 .

Moreover, for the FMA's hand score, only the experimental group showed a statistically significant difference before and after the experiment $(\mathrm{P}<0.05)$. No statistically significant differences were found between the two groups in terms of their therapeutic effects following the intervention $(\mathrm{P}>0.05)$.

Table 3: Comparison of FMA scores between groups

\begin{tabular}{|c|c|c|c|c|c|c|}
\hline & \multicolumn{2}{|c|}{ Experimental group } & \multirow[t]{2}{*}{$\mathbf{p}$} & \multicolumn{2}{|c|}{ Control group } & \multirow[t]{2}{*}{$\mathbf{p}$} \\
\hline & Pre-test & Post-test & & Pre-test & Post-test & \\
\hline FMA Wrist & $4.28 \pm 1.14$ & $6.75 \pm 0.87$ & 0.018 & $4.46 \pm 1.02$ & $5.64 \pm 0.59$ & 0.046 \\
\hline \multirow{3}{*}{$\begin{array}{l}\text { FMA Hand } \\
\text { pre-post }\end{array}$} & \multicolumn{2}{|c|}{$-1.96 \pm 0.23$} & 0.064 & \multicolumn{2}{|c|}{$-1.04 \pm 0.37$} & \\
\hline & \multirow{2}{*}{\multicolumn{2}{|c|}{$-1.44 \pm 0.36$}} & 0.031 & \multicolumn{2}{|c|}{$-0.62 \pm 0.29$} & 0.082 \\
\hline & & & 0.071 & & & \\
\hline
\end{tabular}

$<0.05$, FMA: Fugl-Meyer assessment

\section{Action research ARM test (ARAT)}

The ARAT scores showed a statistically significant difference for both groups before and after the experiment $(\mathrm{P}<0.05)$ as shown in Table 4. Meanwhile, a comparison of the two groups in their therapeutic effects following the intervention exhibited that the experimental group had a larger statistically significant change than that of the control group after the intervention $(\mathrm{P}<0.05)$.

Table 4: Comparison of ARAT scores between groups

\begin{tabular}{|c|c|c|c|c|c|c|}
\hline & \multicolumn{2}{|c|}{ Experimental group } & \multirow[t]{2}{*}{$\mathbf{p}$} & \multicolumn{2}{|c|}{ Control group } & \multirow[t]{2}{*}{$\mathbf{p}$} \\
\hline & Pre-test & Post-test & & Pre-test & Post-test & \\
\hline ARAT & $21.47 \pm 6.31$ & $34 \cdot 55 \pm 7.19$ & 0.000 & $22.37 \pm 7.12$ & $32.97 \pm 5.67$ & 0.000 \\
\hline \multirow[t]{2}{*}{ pre-post } & \multicolumn{2}{|c|}{$-12.48 \pm 3 \cdot 35$} & \multicolumn{4}{|c|}{$-10.69 \pm 4.98$} \\
\hline & & & 0.000 & & & \\
\hline
\end{tabular}

$<0.05$, ARAT: action research arm test

\section{Korean version of the modified barthel index (K-MBI)}

Both groups showed a statistically significant difference in their K-MBI scores before and after the experiment $(\mathrm{P}<0.05)$ as shown in Table 5. In a comparison of the two groups regarding their therapeutic effects after the intervention, the experimental group exhibited a larger statistically significant change than that of the control group $(\mathrm{P}<0.05)$.

Table 5: Comparison of MBI scores between groups

\begin{tabular}{|c|c|c|c|c|c|c|}
\hline & \multicolumn{2}{|c|}{ Experimental group } & \multirow[t]{2}{*}{$\mathbf{p}$} & \multicolumn{2}{|c|}{ Control group } & \multirow[t]{2}{*}{$\mathbf{p}$} \\
\hline & Pre-test & Post-test & & Pre-test & Post-test & \\
\hline MBI & $45.89 \pm 8.95$ & $52.35 \pm 9.19$ & 0.000 & $43.87 \pm 7.55$ & $50.32 \pm 7.48$ & 0.000 \\
\hline pre-post & \multicolumn{2}{|c|}{$-7 \cdot 33 \pm 3.78$} & \multicolumn{4}{|c|}{$-6.02 \pm 3.01$} \\
\hline
\end{tabular}

<0.05, MBI: modified Barthel index 


\section{DISCUSSION}

Stroke is classified as obstructive stroke (in which ischemia attributed to infarction causes hypoxia and brain tissue necrosis) and hemorrhagic stroke (caused by intracranial haemorrhage). Stroke is a primary cause of death and disabilities throughout the world, and its incidence is gradually increasing. Most of the patients develop long-term disabilities due to a chronic lack of exercise associated with a lowered quality of life. This condition is more common among the elderly, but nowadays, stroke also often occurs in those aged 20 to 40 because of changes in the social environment.

The development of medical technology has increased the survival rate of stroke patients, but $70 \%$ to $75 \%$ of them are restricted in performing ADLs due to aftereffects. Over $85 \%$ of stroke patients experience hemiplegia, and over $69 \%$ of hemiplegic patients develop motor disturbance. Although most stroke patients continue to use their affected lower limbs through walking, their upper limbs rely on the unaffected side. As a result, the affected upper limb is less frequently used, thereby worsening functional restrictions with time. Particularly, because stroke patients' independence in performing ADLs depends on the functional recovery of their upper limbs, they should receive therapeutic interventions that can facilitate the upper limb's functional activity. Without proper treatment, $55 \%$ to $75 \%$ of patients exhibit upper limb disorders even six months after stroke onset.

Actual motor disorders that occur in a stroke patient's affected upper limb after this pathological process can be explained by the occurrence of "learned nonuse". Even a patient with a high motor recovery level at an initial evaluation learns the compensation of the unaffected upper limb in the early stages of functional training unless they spontaneously use the affected upper limb. Even with the subsequent restoration of the patient's motor and sensory functions, their learned nonuse of the hand on the affected side results in motor disturbance.

Stroke patients' upper limb problems include spasticity, weakened muscle strength, imbalance, muscle tone changes, arthrochalasis and dystaxia, and sensory disorders, and these losses of motor control substantially confine their independently performed activities, such as ADLs. Also, such restrictions of independent activities in stroke patients lower their quality of life by changing their life patterns toward dependence. In other words, brain tissue damage deforms muscle activity, reduces muscular strength and contraction, and reduces the ability for motor control and planning and the integration of sensory information, thereby eventually constraining patients' selective movements.

The facilitation of stroke patients' rehabilitation and return to their daily lives requires therapeutic interventions that can reduce the long-term nonuse, incompetence, and functional impairment of their upper limbs. In clinical practice, therapeutic approaches using traditional methods, such as PNF, Bobath therapy, and the Brunnstrom approach, are frequently applied to restore upper limb function. Additional methods include CIMT, which encourages the use of the affected upper limb while restricting the use of the unaffected upper limb, feedback control therapy through mirror images using robot-assisted systems, the treatment that applies feedback training to movements on the affected side using a mirror, treatment using quick tempos, and aquatic therapy applied via buoyance to patients with spasticity and weak muscular strength. Also, studies using overlapping therapeutic effects are underway to improve upper limb functions, including electric stimulation therapy and NDT, taping and NDT, elastic bands and NDT, and aids and taping.

In this study, the overlapping effects of ESWT and PNF on stroke patients' improvement in upper limb function were investigated with ESWT as an electrical stimulation therapy before applying PNF. PNF is a therapeutic approach for rehabilitating stroke patients that facilitates the response of nerve roots by stimulating the proprioceptor. As a mass movement using diagonal and spiral exercise patterns, PNF induces muscle contraction on the weak side by promoting interactions between the body's two sides and training the strong side via resistance exercises. PNF's diagonal and spiral exercise patterns are very similar to movements generated by functional activities. Mass movement patterns can be categorized as the upper limb, lower limb, trunk, head, and mandibular patterns. These patterns evolve into more diverse patterns with joints that serve as axes around which major movements occur.

PNF stimulates the proprioceptor inside the muscle and tendon, thereby enhancing their functions and increasing muscular strength, flexibility, and equilibrium. Also, according to PNF's approach, resistance and traction can increase muscular strength by causing muscle contraction and offer stability to the area that generates movements by controlling the direction, size, and speed of movements. By stimulating the proprioceptor and facilitating normal responses using spiral patterns, PNF can promote the response of nerve roots via their mechanism by stimulating proprioception in the muscle spindle that discharges afferent excitation in response to muscle length or tension.

ESWT has been used orthopedically since its first adoption as a treatment for kidney stones in the early 1980s. In recent years, it has gradually expanded as a noninvasive pain and rehabilitation therapy for musculoskeletal diseases. ESWT is categorized as focused and radial types according to the principle of shockwave generation. The focused type collects shock waves from the site of their generation into a single location through collection plates. The radial type gradually weakens shock waves with increases in distance due to its 
dispersing pattern. Currently, available focused-type ESWT equipment's shock wave generation devices are classified as electro-hydraulic, electromagnetic, and piezoelectric types.

ESWT is used for diseases such as plantar fasciitis, lateral epicondylitis, patellar tendinitis, nonunion of the iliac fracture, and osteonecrosis of the femoral head. It reduces pain. helps regenerate impaired tendons and bones and facilitates blood supply in patients with diabetic foot ulceration and ischemic heart disease. Previous research has found that ESWT promotes angiopoiesis and induces the formation of lymphatic vessels through the upregulation of the vascular endothelial growth factor (VEGF) and the basic fibroblast growth factor (bFGF). A subsequent study on patients with secondary lymphedema proved the therapeutic effects of ESWT. Accordingly, ESWT is endorsed as a noninvasive alternative treatment method for patients with lymphedema. ESWT enhances cytopermeability via a mechanical force generated by acoustic shock waves and increases the expression of factors involved in the formation of lymphatic vessels. It also increases skin elasticity and facilitates the organization of the skin structure.

In this study, low-intensity ESWT was employed to examine stroke patients' functional improvement through reduced spasticity. In terms of muscle tone and FMA scores for the extension of the elbow joint, the experimental group treated with ESWT showed a larger reduction in spasticity and a higher increase in the FMA score than those of the control group. However, no statistically significant differences were observed between the two groups. A study (Taheri et al., 2017) focusing on 28 stroke patients with spasticity reported that the experimental group, which was treated with ESWT once a week for three weeks along with the administration of anticonvulsants and stretching exercises, made statistically significant improvements in the Modified Ashworth Scale (MAS), pain, and ROM compared with the control group, which only received anticonvulsants and performed stretching exercises.

In a study (Wu et al., 2018) that investigated differences in the therapeutic effects of ESWT and a treatment based on botulinum toxin types, the Extracorporeal Shock-Wave Therapy (ESWT) group achieved statistically significant improvements in the modified Ashworth scale (MAS) and Fugl-Meyer assessment (FMA) scores compared with those of the control group. The present study did not obtain the same results as those of these prior studies, which may be because it failed to maximize the effects of functional improvements in the hands and wrists in terms of shock wave generation methods and their application site and time.

\section{CONCLUSION}

The present study's findings indicated differences between the two groups in the ARAT and ADL evaluation. The ex- perimental group, which was treated with ESWT before PNF, produced larger statistically significant increases in the ARAT and K-MBI than those of the control group. According to a study (Guo et al., 2019) that explored the overlapping effects of ESWT and mirror therapy in 120 stroke patients, a group that received both therapies reduced their spasticity and improved their upper limb function more effectively than a group treated with only one therapy. This outcome is probably because the application of ESWT increased patients' independence in performing ADLs by reducing the limitations in their functional activity through spasticity control.

The present study has some limitations. It had a small number of subjects and a short period of therapeutic interventions. Also, these therapies were not applied from the early stages of the disease. Moreover, their therapeutic effects with time were not evaluated. Nevertheless, this study may promote new studies that compare the effects of therapies for stroke through their applications in the early stages of the disease based on varied body regions, treatment durations, and quantitative evaluations.

\section{ACKNOWLEDGMENTS}

This research was supported by Kyungdong University Research Fund, 2020.

\section{Conflict of Interest: Nil}

Funding Source: Kyungdong University Research Fund, 2020

\section{REFERENCES}

1. Gittler M, Davis AM. Guidelines for adult stroke rehabilitation and recovery. Jama. 2018 Feb 27;319(8):820-1.

2. Pérez-Cruzado D, Merchán Baeza JA, González-Sánchez M, Cuesta Vargas AI. Systematic review of mirror therapy compared with conventional rehabilitation in upper extremity function in stroke survivors. Australian occupational therapy journal. $2017 ; 64(2): 91-112$.

3. Schneider EJ, Lannin NA, Ada L, Schmidt J. Increasing the amount of usual rehabilitation improves activity after stroke: a systematic review. Journal of physiotherapy. 2016 1;62(4):1827.

4. Yew KS, Cheng EM. Diagnosis of acute stroke. American family physician. 2015 Apr 15;91(8):528-36.

5. Törnbom K, Persson HC, Lundälv J, Sunnerhagen KS. The impact of physical function on participation in the first year poststroke. Acta neurologica Scandinavica. 2017 ;135(6):649-55.

6. Koh SH, Park HH. Neurogenesis in stroke recovery. Translational stroke research. $2017 \mathrm{Feb}$ 1;8(1):3-13.

7. Campbell BC. Thrombolysis and thrombectomy for acute ischemic stroke: strengths and synergies. In Seminars in thrombosis and hemostasis 2017; 43( 02): 185-190).

8. Kiper P, Szczudlik A, Agostini M, Opara J, Nowobilski R, Ventura L, Tonin P, Turolla A. Virtual reality for upper limb re- 
habilitation in subacute and chronic stroke: a randomized controlled trial. Archives of physical medicine and rehabilitation. $2018 ; 99(5): 834-42$.

9. Jung K, Kim Y, Chung Y, Hwang S. Weight-shift training improves trunk control, proprioception, and balance in patients with chronic hemiparetic stroke. The Tohoku journal of experimental medicine. 2014;232(3):195-9.

10. Rand D. Mobility, balance and balance confidence-correlations with daily living of individuals with and without mild proprioception deficits post-stroke. NeuroRehabilitation. 2018 Jan $1 ; 43(2): 219-26$.

11. Morreale M, Marchione P, Pili A, Lauta A, Castiglia SF, Spallone A, Pierelli F, Giacomini P. Early versus delayed rehabilitation treatment in hemiplegic patients with ischemic stroke: proprioceptive or cognitive approach. Eur J Phys Rehabil Med. 2016 ;52(1):81-9.

12. An M, Shaughnessy M. The effects of exercise-based rehabilitation on balance and gait for stroke patients: a systematic review. Journal of neuroscience nursing. 2011;43(6):298-307.

13. Murphy SA, Negro F, Farina D, Onushko T, Durand M, Hunter $\mathrm{SK}$, Schmit BD, Hyngstrom A. Stroke increases ischemia-related decreases in motor unit discharge rates. Journal of Neurophysiology. 2018 Dec 1;120(6):3246-56.

14. El-Helow MR, Zamzam ML, Fathalla MM, El-Badawy MA, El Nahhas N, El-Nabil LM, Awad MR, Von Wild K. Efficacy of modified constraint-induced movement therapy in acute stroke. Eur J Phys Rehabil Med. 2015;51(4):371-9.

15. Sharififar S, Shuster JJ, Bishop MD. Adding electrical stimulation during standard rehabilitation after stroke to improve motor function. A systematic review and meta-analysis. Annals of physical and rehabilitation medicine. 2018 Sep 1;61(5):339-44.

16. Huisstede BM, Gebremariam L, van der Sande R, Hay EM, Koes BW. Evidence for effectiveness of Extracorporal ShockWave Therapy (ESWT) to treat calcific and non-calcific rotator cuff tendinosis-a systematic review. Manual therapy. 2011 ;16(5):419-33.

17. Wang CJ, Cheng JH, Chou WY, Hsu SL, Chen JH, Huang CY. Changes of articular cartilage and subchondral bone after extracorporeal shockwave therapy in osteoarthritis of the knee. International journal of medical sciences. 2017;14(3):213.

18. Nelson ML, McKellar KA, Yi J, Kelloway L, Munce S, Cott C, Hall R, Fortin M, Teasell R, Lyons R. Stroke rehabilitation evidence and comorbidity: a systematic scoping review of randomized controlled trials. Topics in stroke rehabilitation. 2017 ;24(5):374-80.

19. Pistoia F, Sacco S, Degan D, Tiseo C, Ornello R, Carolei A. Hypertension and stroke: epidemiological aspects and clinical evaluation. High Blood Pressure \& Cardiovascular Prevention. 2016 ;23(1):9-18.

20. Krakauer JW. Motor learning: its relevance to stroke recovery and neurorehabilitation. Current opinion in neurology. 2006 ;19(1):84-90.

21. Veerbeek JM, Langbroek-Amersfoort AC, Van Wegen EE, Meskers CG, Kwakkel G. Effects of robot-assisted therapy for the upper limb after stroke: a systematic review and meta-analysis. Neurorehabilitation and neural repair. 2017 Feb;31(2):10721.

22. Arwert H, Schut S, Boiten J, Vliet Vlieland T, Meesters J. Patient reported outcomes of hand function three years after stroke. Topics in sTroke rehabiliTaTion. $2018 ; 25(1): 13-9$.

23. Veldema J, Bösl K, Nowak DA. Cortico-spinal excitability and hand motor recovery in stroke: a longitudinal study. Journal of neurology. 2018 May 1;265(5):1071-8.
24. Ribeiro TS, Silva WH, de Alencar Caldas VV, Silva DL, Cavalcanti FA, Lindquist AR. Effects of a training program based on the proprioceptive neuromuscular facilitation method on poststroke motor recovery: a preliminary study. Journal of bodywork and movement therapies. 2014;18(4):526-32.

25. Kwakkel G, Veerbeek JM, van Wegen EE, Wolf SL. Constraintinduced movement therapy after stroke. The Lancet Neurology. 2015;14(2):224-34.

26. Arya KN, Pandian S, Puri V. Mirror illusion for sensori-motor training in stroke: a randomized controlled trial. Journal of Stroke and Cerebrovascular Diseases. 2018 ;27(11):3236-46.

27. Choi YK, Nam CW, Lee JH, Park YH. The effects of taping prior to PNF treatment on lower extremity proprioception of hemiplegic patients. Journal of physical therapy science. 2013;25(9):1119-22.

28. Gunning E, Uszynski MK. Effectiveness of the proprioceptive neuromuscular facilitation method on gait parameters in patients with stroke: a systematic review. Archives of physical medicine and rehabilitation. 2019 May 1;100(5):980-6.

29. Ribeiro T, Britto H, Oliveira D, Silva E, Galvao E, Lindquist A. Effects of treadmill training with partial body weight support and the proprioceptive neuromuscular facilitation method on hemiparetic gait: a randomized controlled study. Eur J Phys Rehabil Med. 2013;49(4):451-61.

30. Al-Mendalawi MD. Fulminant subacute sclerosing panencephalitis in an immunized 20-month-old Indian boy. Neurosciences. 2019 Jan 1;24(1).

31. Gruenwald I, Kitrey ND, Appel B, Vardi Y. Low Intensity Extracorporeal Shock Wave Therapy in Vascular Disease and Erectile Dysfunction: Theory and Outcomes. Sexual medicine reviews. 20131;1(2):83-90.

32. Roehrig GJ, Baumhauer J, DiGiovanni BF, Flemister AS. The role of extracorporeal shock wave on plantar fasciitis. Foot and ankle clinics. 2005;10(4):699-712.

33. E. J. Kim. Effect of relationships with parents, friends, and selfesteem on academic performance and delinquency of middle school students. International Journal of Child Warfare Promotion and Management. 2018;2(2):75-80.

34. Shi L, Gao F, Sun W, Wang B, Guo W, Cheng L, Li Z, Wang W. Short-term effects of extracorporeal shock wave therapy on bone mineral density in postmenopausal osteoporotic patients. Osteoporosis International. 2017;28(10):2945-53.

35. Y. P. Kim \& J. G. Kang. The Study on Characteristics in Articles related to the Parents of Children with Autism Spectrum Disorders in South Korea. International Journal of Child Warfare Promotion and Management. 2018;2(2):51-56.

36. Kim IG, Lee JY, Lee DS, Kwon JY, Hwang JH. Extracorporeal shock wave therapy combined with vascular endothelial growth factor-C hydrogel for lymphangiogenesis. Journal of Vascular Research. 2013;50(2):124-33.

37. J. H. Lee. Verification of Overlapping Effects of Proprioceptive Training and Electrical Stimulation Therapy on Reducing Upper Limb Spasticity and Improving Physical Functions and Activities of Daily Living. International Journal of Social Welfare Promotion and Management. 2020;7(2):33-38.

38. Chun Y. A Study of Perceived Parental Faith-nurturing Activities and Children's Psychological Well-being. 2019;3(1):3

39. Taheri P, Vahdatpour B, Mellat M, Ashtari F, Akbari M. Effect of extracorporeal shock wave therapy on lower limb spasticity in stroke patients. Archives of Iranian medicine. 2017 ;20(6):33843.

40. Wu YT, Yu HK, Chen LR, Chang CN, Chen YM, Hu GC. Extracorporeal shock waves versus botulinum toxin type A in the 
treatment of poststroke upper limb spasticity: a randomized noninferiority trial. Archives of physical medicine and rehabilitation. 2018 ;99(11):2143-50.

41. Guo J, Qian S, Wang Y, Xu A. Clinical study of combined mirror and extracorporeal shock wave therapy on upper limb spasticity in poststroke patients. International journal of rehabilitation research. Internationale Zeitschrift fur Rehabilitationsforschung. Revue internationale de recherches de readaptation. 2019;42(1):31. 